International Journal of Pure and Applied Mathematics

Volume 89 No. 2 2013, 215-224

ISSN: 1311-8080 (printed version); ISSN: 1314-3395 (on-line version)

url: http://www.ijpam.eu

doi: http://dx.doi.org/10.12732/ijpam.v89i2.7

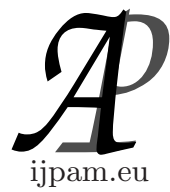

\title{
GEOMETRICAL COUPLING FIELDS OF \\ A HYPERCOMPLEX TYPE
}

\author{
J.A.P.F. Marão ${ }^{1}$, M.F. Borges ${ }^{2} \S$ \\ ${ }^{1}$ Department of Mathematics \\ Federal University of Maranhão - São Luís-MA \\ 65085-580, Maranhão, BRAZIL \\ ${ }^{2}$ UNESP - São Paulo State University \\ S.J. Rio Preto Campus \\ 15054-000, São José do Rio Preto, BRAZIL
}

\begin{abstract}
This paper presents an application of Laplace's equation obtained from a quaternionic function that satisfies the Cauchy-Riemann conditions determined earlier by Borges and Machado [1]. Therefore, we show that it is possible to express in a single equation gravity, electric and magnetic potential fields, and this expression can only be provided due to a function that will be called here the coupling function.
\end{abstract}

AMS Subject Classification: 30G99, 30E99

Key Words: quaternions, Laplace's equations, geoemtrical coupling fields

\section{Introduction}

The ability to express the gravitational, electric and magnetic potentials in a single equations has not been explored substantially in the context of hypercomplex geometry. However, there is a case of time-independent coupling in [2], in which the Poisson equation for both electric and gravitational potentials generating a coupling equations between both fields.

Received: August 26, 2013

(C) 2013 Academic Publications, Ltd.

$\S$ Correspondence author url: www.acadpubl.eu 
The problem of determining electrical, gravitational and magnetic coupling potential field equations in time depending will be the object of this work. So far one of the main motivations and the starting point of our investigation is the law of gravitation due to Gauss, which is already well known from classical physics. This law in Gauss form, explains gravitation in terms of a gravitational and potential:

$$
\nabla^{2} \phi(t, x, y, z)=4 \pi G \rho(t, x, y, z)
$$

The law of gravitation to Gauss: $\phi(t, x, y, z)$ is the gravitational potential $G$ a gravitational constant and $\rho(t, x, y, z)$ the density.

Other classical equations as equation (2) mentioned below will be important for our scope of searching for a coupling theory in a hypercomplex context and involving gravitational, electrical and magnetic fields.

For instance:

$$
\begin{aligned}
-\frac{1}{c^{2}} \frac{\partial^{2} \varphi}{\partial t^{2}}(t, x, y, z)+\frac{\partial^{2} \varphi}{\partial x^{2}}(t, x, y, z)+\frac{\partial^{2} \varphi}{\partial y^{2}}(t, x, y, z) & +\frac{\partial^{2} \varphi}{\partial z^{2}}(t, x, y, z) \\
& =-4 \pi \rho_{e}(t, x, y, z)
\end{aligned}
$$

where $c$ is the speed of light, $\varphi$ is the electric potential and $\rho_{e}$ is called the charge density.

The equations (2) and (3) have in common a number of variables, and a function regarded as potential. However it can not be framed in the Laplace like equation in four dimensions, as it is shown in [3], which is given by:

$$
\sum_{i=1}^{4} \frac{\partial^{2} F}{\partial x_{i}^{2}}=0, \quad i=1,2,3,4
$$

where $F$ is real function of variables $t, x, y, z$.

\section{Obtaining the Laplace's Equations.}

Let us considering $f(q)$ - ( $q$ being a quaternion number $)$ - a quaternionic function. We can associate with it a set of equations called the Cauchy-Riemann like relations [1]. Firstly, the well known theorem to a function of a complex variables is presented below:

Theorem 1. If $f(z)=u(x, y)+i v(x, y)$ a function defined and continues in a neighborhood of the point $z=x+i y$ and differenciable in $z$. Then the 
partial derivatives of the first order of $u(x, y)$ and $v(x, y)$ exist and satisfy the relations:

$$
\frac{\partial u}{\partial x}(x, y)=\frac{\partial v}{\partial y}(x, y)
$$

and

$$
\frac{\partial u}{\partial y}(x, y)=-\frac{\partial v}{\partial x}(x, y)
$$

The set of equations (4) and (5) are called Cauchy-Riemann relations and are well known from the classical literature [4]. If the set of functions above is class of $C^{2}$, then they satisfy the Schwartz's theorem, then it follows that:

$$
\nabla^{2} u(x, y)=0
$$

and

$$
\nabla^{2} v(x, y)=0 .
$$

With these results, it is possible to generalize the last two equations for the case of a quaternionic function as seen in [3] and [1].

Theorem 2. For any pair points $a$ and $b$ and any path joining them simply conect subdomain of the four-dimmensional space, the integral $\int_{a}^{b} f d q$ is independent form the given path if and only if there is a function $F=$ $F_{1}+F_{2} i+F_{3} j+F_{4} k$ such that $\int_{a}^{b} f d q=F(a)-F(b)$, and satisfying the following relations:

$$
\begin{gathered}
\frac{\partial F_{1}}{\partial x_{1}}=\frac{\partial F_{2}}{\partial x_{2}}=\frac{\partial F_{3}}{\partial x_{3}}=\frac{\partial F_{4}}{\partial x_{4}} \\
\frac{\partial F_{2}}{\partial x_{1}}=-\frac{\partial F_{1}}{\partial x_{2}}=-\frac{\partial F_{3}}{\partial x_{4}}=\frac{\partial F_{4}}{\partial x_{3}} \\
\frac{\partial F_{3}}{\partial x_{1}}=-\frac{\partial F_{1}}{\partial x_{3}}=-\frac{\partial F_{2}}{\partial x_{4}}=\frac{\partial F_{4}}{\partial x_{2}} \\
\frac{\partial F_{4}}{\partial x_{1}}=\frac{\partial F_{1}}{\partial x_{4}}=-\frac{\partial F_{2}}{\partial x_{3}}=-\frac{\partial F_{3}}{\partial x_{2}}
\end{gathered}
$$

Proof. The proof of this theorem can be analyzed in greater detail in [1].

Now, let us consider the derivation of the above (7), (8), (9) and (10) admitting that the real functions in question are of class $C^{2}$. The followings equations are obtained [3]:

$$
\frac{\partial F_{1}}{\partial x_{1}^{2}}+\frac{\partial F_{1}}{\partial x_{2}^{2}}+\frac{\partial F_{1}}{\partial x_{3}^{2}}+\frac{\partial F_{1}}{\partial x_{4}^{2}}=0
$$




$$
\begin{aligned}
& \frac{\partial F_{2}}{\partial x_{1}^{2}}+\frac{\partial F_{2}}{\partial x_{2}^{2}}+\frac{\partial F_{2}}{\partial x_{3}^{2}}+\frac{\partial F_{2}}{\partial x_{4}^{2}}=0 \\
& \frac{\partial F_{3}}{\partial x_{1}^{2}}+\frac{\partial F_{3}}{\partial x_{2}^{2}}+\frac{\partial F_{3}}{\partial x_{3}^{2}}+\frac{\partial F_{3}}{\partial x_{4}^{2}}=0
\end{aligned}
$$

and

$$
\frac{\partial F_{4}}{\partial x_{1}^{2}}+\frac{\partial F_{4}}{\partial x_{2}^{2}}+\frac{\partial F_{4}}{\partial x_{3}^{2}}+\frac{\partial F_{4}}{\partial x_{4}^{2}}=0
$$

Theorem 3. If $G$ is an function of $t, x, y$ e $z$, variables, then the transformation of $t$ for $t^{\prime}$, such that

$$
\frac{d t}{d t^{\prime}}=1
$$

of temporal coordinates (translation for sample), satisfies the following equation:

$$
\frac{\partial^{2} G}{\partial t^{2}}(t, x, y, z)=\frac{\partial^{2} G^{\prime}}{\partial t^{\prime 2}}\left(t^{\prime}, x, y, z\right)
$$

where $G^{\prime}$ is the $G$ applied at time $t^{\prime}$ for the same spatial coordinates $x, y$ e $z$.

Proof. Initially:

$$
\frac{\partial G}{\partial t}(t, x, y, z)=\frac{\partial G}{\partial t^{\prime}}\left(t^{\prime}, x, y, z\right) \frac{d t^{\prime}}{d t}=\frac{\partial G}{\partial t^{\prime}}\left(t^{\prime}, x, y, z\right)
$$

The derivative is given for:

$$
\frac{\partial^{2} G}{\partial t^{2}}(t, x, y, z)=\frac{\partial^{2} G^{\prime}}{\partial t^{\prime 2}}\left(t^{\prime}, x, y, z\right) \frac{d t^{\prime}}{d t}=\frac{\partial^{2} G^{\prime}}{\partial t^{\prime 2}}\left(t^{\prime}, x, y, z\right)
$$

Note. For mixed derivatives with respect to time and spatial coordinates $x, y$ or $z$, the transformation $t$ for $t$ is easily obtained according to the following calculation for a hypothetical situation:

$$
\begin{aligned}
\frac{\partial^{2} G}{\partial t \partial x}(t, x, y, z)=\frac{\partial}{\partial t}\left(\frac{\partial G}{\partial x}\right)(t, x, y, z)=\frac{\partial}{\partial t^{\prime}}\left(\frac{\partial G^{\prime}}{\partial x}\right)\left(t^{\prime}, x, y, z\right) \frac{d t^{\prime}}{d t} & \\
& =\frac{\partial^{2} G^{\prime}}{\partial t^{\prime} \partial x}\left(t^{\prime}, x, y, z\right) .
\end{aligned}
$$

Seeking to adapt the equations given in (1) and (2) to Laplace's equation obtained earlier, it will be used the following change in the configuration of derivatives against time. After translating the coordinates in second derivative 
of $F(t, x, y, z)$ relative to $t$ it is performed the second derivative of $F^{\prime}\left(t^{\prime}, x, y, z\right)$ relative to $t^{\prime}$ plus a function $\psi\left(t^{\prime}, x, y, z\right)$ as follows:

$$
\frac{\partial^{2} F}{\partial t^{2}}(t, x, y, z)=\frac{\partial^{2} F^{\prime}}{\partial t^{\prime 2}}\left(t^{\prime}, x, y, z\right)+\psi\left(t^{\prime}, x, y, z\right) .
$$

\section{Transformations in Laplace's Equations.}

Let us consider, $f(q)=F_{1}(t, x, y, z)+F_{2}(t, x, y, z) i+F_{3}(t, x, y, z) j+F_{4}(t, x, y, z) k$ that satisfies the Cauchy-Riemann conditions.

$$
\begin{gathered}
\frac{\partial F_{1}}{\partial t}=\frac{\partial F_{2}}{\partial x}=\frac{\partial F_{3}}{\partial y}=\frac{\partial F_{4}}{\partial z} \\
\frac{\partial F_{2}}{\partial t}=-\frac{\partial F_{1}}{\partial x}=-\frac{\partial F_{3}}{\partial z}=\frac{\partial F_{4}}{\partial y} \\
\frac{\partial F_{3}}{\partial t}=-\frac{\partial F_{1}}{\partial y}=-\frac{\partial F_{2}}{\partial z}=\frac{\partial F_{4}}{\partial x} \\
\frac{\partial F_{4}}{\partial t}=\frac{\partial F_{1}}{\partial z}=-\frac{\partial F_{2}}{\partial y}=-\frac{\partial F_{3}}{\partial x}
\end{gathered}
$$

Deriving the conditions of equation (17), we have that:

$$
\begin{aligned}
& \frac{\partial^{2} F_{1}}{\partial t^{2}}=\frac{\partial^{2} F_{2}}{\partial t \partial x}=\frac{\partial^{2} F_{3}}{\partial t \partial y}=\frac{\partial^{2} F_{4}}{\partial t \partial z} \\
& \frac{\partial^{2} F_{1}}{\partial t \partial x}=\frac{\partial^{2} F_{2}}{\partial x^{2}}=\frac{\partial^{2} F_{3}}{\partial x \partial y}=\frac{\partial^{2} F_{4}}{\partial x \partial z} \\
& \frac{\partial^{2} F_{1}}{\partial y \partial t}=\frac{\partial^{2} F_{2}}{\partial y \partial x}=\frac{\partial^{2} F_{3}}{\partial y^{2}}=\frac{\partial^{2} F_{4}}{\partial z \partial y} \\
& \frac{\partial^{2} F_{1}}{\partial t \partial z}=\frac{\partial^{2} F_{2}}{\partial z \partial x}=\frac{\partial^{2} F_{3}}{\partial z \partial y}=\frac{\partial^{2} F_{4}}{\partial z^{2}} .
\end{aligned}
$$

Deriving the conditions of equation (18), we have:

$$
\begin{aligned}
& \frac{\partial^{2} F_{2}}{\partial t^{2}}=-\frac{\partial^{2} F_{1}}{\partial t \partial x}=-\frac{\partial^{2} F_{3}}{\partial t \partial z}=\frac{\partial^{2} F_{4}}{\partial t \partial y} \\
& \frac{\partial^{2} F_{2}}{\partial t \partial x}=-\frac{\partial^{2} F_{1}}{\partial x^{2}}=-\frac{\partial^{2} F_{3}}{\partial x \partial z}=\frac{\partial^{2} F_{4}}{\partial y \partial x} \\
& \frac{\partial^{2} F_{2}}{\partial y \partial t}=-\frac{\partial^{2} F_{1}}{\partial y \partial x}=-\frac{\partial^{2} F_{3}}{\partial y \partial z}=\frac{\partial^{2} F_{4}}{\partial y^{2}} \\
& \frac{\partial^{2} F_{2}}{\partial z \partial t}=-\frac{\partial^{2} F_{1}}{\partial z \partial x}=-\frac{\partial^{2} F_{3}}{\partial z^{2}}=\frac{\partial^{2} F_{4}}{\partial z \partial y} .
\end{aligned}
$$


Deriving the conditions of equation (19), we obtain:

$$
\begin{aligned}
& \frac{\partial^{2} F_{3}}{\partial t^{2}}=-\frac{\partial^{2} F_{1}}{\partial t \partial y}=-\frac{\partial^{2} F_{2}}{\partial t \partial z}=\frac{\partial^{2} F_{4}}{\partial t \partial x} \\
& \frac{\partial^{2} F_{3}}{\partial t \partial x}=-\frac{\partial^{2} F_{1}}{\partial x \partial y}=-\frac{\partial^{2} F_{2}}{\partial x \partial z}=\frac{\partial^{2} F_{4}}{\partial x^{2}} \\
& \frac{\partial^{2} F_{3}}{\partial y \partial t}=-\frac{\partial^{2} F_{1}}{\partial y^{2}}=-\frac{\partial^{2} F_{2}}{\partial z \partial y}=\frac{\partial^{2} F_{4}}{\partial y \partial x} \\
& \frac{\partial^{2} F_{3}}{\partial t \partial z}=-\frac{\partial^{2} F_{1}}{\partial z \partial y}=-\frac{\partial^{2} F_{2}}{\partial z^{2}}=\frac{\partial^{2} F_{4}}{\partial z \partial x} .
\end{aligned}
$$

And finally, in deriving the conditions of equation (20), it follows that:

$$
\begin{aligned}
& \frac{\partial^{2} F_{4}}{\partial t^{2}}=\frac{\partial^{2} F_{1}}{\partial t \partial z}=-\frac{\partial^{2} F_{2}}{\partial t \partial y}=-\frac{\partial^{2} F_{3}}{\partial t \partial x} \\
& \frac{\partial^{2} F_{4}}{\partial t \partial x}=\frac{\partial^{2} F_{1}}{\partial x \partial z}=-\frac{\partial^{2} F_{2}}{\partial x \partial y}=-\frac{\partial^{2} F_{3}}{\partial x^{2}} \\
& \frac{\partial^{2} F_{4}}{\partial y \partial t}=\frac{\partial^{2} F_{1}}{\partial y \partial z}=-\frac{\partial^{2} F_{2}}{\partial y^{2}}=-\frac{\partial^{2} F_{3}}{\partial y \partial x} \\
& \frac{\partial^{2} F_{4}}{\partial t \partial z}=\frac{\partial^{2} F_{1}}{\partial z^{2}}=-\frac{\partial^{2} F_{2}}{\partial z \partial y}=-\frac{\partial^{2} F_{3}}{\partial z \partial x} .
\end{aligned}
$$

Laplace's equations obtained here have no physical meaning yet. Therefore, the equations may have physical significance using the principle of transformation $t$ for $t^{\prime}$ plus function $\varphi$, and this will be done on each of the equations (1) and (2) as follows:

$$
\frac{\partial F_{2}}{\partial t^{2}}+\frac{\partial F_{2}}{\partial x^{2}}+\frac{\partial F_{2}}{\partial y^{2}}+\frac{\partial F_{2}}{\partial z^{2}}=0
$$

Making in (16) the transformation and associating the function $\psi\left(t^{\prime}, x, y, z\right)$ to $4 \pi G \rho\left(t^{\prime}, x, y, z\right)$, it generates a new $F^{\prime}$ as follows:

$$
\begin{array}{r}
{\left[\frac{\partial^{2} F_{2}^{\prime}}{\partial t^{\prime 2}}\left(t^{\prime}, x, y, z\right)-4 \pi G \rho\left(t^{\prime}, x, y, z\right)\right]+\frac{\partial^{2} F_{2}^{\prime}}{\partial x^{2}}(t, x, y, z)+\frac{\partial^{2} F_{2}^{\prime}}{\partial y^{2}}(t, x, y, z)} \\
+\frac{\partial^{2} F_{2}^{\prime}}{\partial z^{2}}\left(t^{\prime}, x, y, z\right)=0
\end{array}
$$

this equation represents a Gauss-like law for gravitation.

$$
\frac{\partial F_{3}}{\partial t^{2}}+\frac{\partial F_{3}}{\partial x^{2}}+\frac{\partial F_{3}}{\partial y^{2}}+\frac{\partial F_{3}}{\partial z^{2}}=0
$$


Applying the transformation in the equation it follows that:

$$
\frac{\partial^{2} F_{3}}{\partial t^{2}}(t, x, y, z)=-\frac{1}{c^{2}} \frac{\partial^{2} F_{3}^{\prime}}{\partial t^{\prime}}\left(t^{\prime}, x, y, z\right)+4 \pi \rho_{e}\left(t^{\prime}, x, y, z\right)
$$

which generating the following equation:

$$
\begin{array}{r}
{\left[-\frac{1}{c^{2}} \frac{\partial^{2} F_{3}^{\prime}}{\partial t^{2}}(t, x, y, z)+4 \pi \rho_{e}(t, x, y, z)\right]+\frac{\partial^{2} F_{3}^{\prime}}{\partial x^{2}}(t, x, y, z)+\frac{\partial^{2} F_{3}^{\prime}}{\partial y^{2}}(t, x, y, z)} \\
+\frac{\partial^{2} F_{3}^{\prime}}{\partial z^{2}}(t, x, y, z)=0, \quad
\end{array}
$$

which is the equation for the electrical potential.

Comparing the equations (26) and (28), after transformation $t$ for $t^{\prime}$, we obtain the following sets:

$$
\begin{aligned}
& \frac{\partial^{2} F_{1}^{\prime}}{\partial t^{\prime 2}}=\frac{\partial^{2} F_{2}^{\prime}}{\partial t^{\prime} \partial x}=\frac{\partial^{2} F_{3}^{\prime}}{\partial t^{\prime} \partial y}=\frac{\partial^{2} F_{4}^{\prime}}{\partial t^{\prime} \partial z} \\
& \frac{\partial^{2} F_{1}^{\prime}}{\partial t^{\prime} \partial x}=\frac{\partial^{2} F_{2}^{\prime}}{\partial x^{2}}=\frac{\partial^{2} F_{3}}{\partial x \partial y}=\frac{\partial^{2} F_{4}^{\prime}}{\partial x \partial z} \\
& \frac{\partial^{2} F_{1}^{\prime}}{\partial y \partial t^{\prime}}=\frac{\partial^{2} F_{2}^{\prime}}{\partial y \partial x}=\frac{\partial^{2} F_{3}}{\partial y^{2}}=\frac{\partial^{2} F_{4}^{\prime}}{\partial z \partial y} \\
& \frac{\partial^{2} F_{1}^{\prime}}{\partial t^{\prime} \partial z}=\frac{\partial^{2} F_{2}^{\prime}}{\partial z \partial x}=\frac{\partial^{2} F_{3}^{\prime}}{\partial z \partial y}=\frac{\partial^{2} F_{4}}{\partial z^{2}} . \\
& \frac{\partial^{2} F_{2}^{\prime}}{\partial t^{\prime 2}}\left(t^{\prime}, x, y, z\right)-4 \pi G \rho\left(t^{\prime}, x, y, z\right)=-\frac{\partial^{2} F_{1}^{\prime}}{\partial t^{\prime} \partial x}=-\frac{\partial^{2} F_{3}^{\prime}}{\partial t^{\prime} \partial z}=\frac{\partial^{2} F_{4}^{\prime}}{\partial t^{\prime} \partial y} \\
& \frac{\partial^{2} F_{2}^{\prime}}{\partial t^{\prime} \partial x}=-\frac{\partial^{2} F_{1}^{\prime}}{\partial x^{2}}=-\frac{\partial^{2} F_{3}^{\prime}}{\partial x \partial z}=\frac{\partial^{2} F_{4}^{\prime}}{\partial y \partial x} \\
& \frac{\partial^{2} F_{2}^{\prime}}{\partial y \partial t^{\prime}}=-\frac{\partial^{2} F_{1}^{\prime}}{\partial y \partial x}=-\frac{\partial^{2} F_{3}^{\prime}}{\partial y \partial z}=\frac{\partial^{2} F_{4}^{\prime}}{\partial y^{2}} \\
& \frac{\partial^{2} F_{2}^{\prime}}{\partial z \partial t^{\prime}}=-\frac{\partial^{2} F_{1}^{\prime}}{\partial z \partial x}=-\frac{\partial^{2} F_{3}^{\prime}}{\partial z^{2}}=\frac{\partial^{2} F_{4}^{\prime}}{\partial z \partial y} . \\
& -\frac{1}{c^{2}} \frac{\partial^{2} F_{3}^{\prime}}{\partial t^{\prime 2}}\left(t^{\prime}, x, y, z\right)+4 \pi \rho_{e}\left(t^{\prime}, x, y, z\right)=-\frac{\partial^{2} F_{1}^{\prime}}{\partial t^{\prime} \partial y}=-\frac{\partial^{2} F_{2}^{\prime}}{\partial t^{\prime} \partial z}=\frac{\partial^{2} F_{4}^{\prime}}{\partial t^{\prime} \partial x} \\
& \frac{\partial^{2} F_{3}^{\prime}}{\partial t^{\prime} \partial x}=-\frac{\partial^{2} F_{1}^{\prime}}{\partial x \partial y}=-\frac{\partial^{2} F_{2}^{\prime}}{\partial x \partial z}=\frac{\partial^{2} F_{4}^{\prime}}{\partial x^{2}} \\
& \frac{\partial^{2} F_{3}^{\prime}}{\partial y \partial t^{\prime}}=-\frac{\partial^{2} F_{1}^{\prime}}{\partial y^{2}}=-\frac{\partial^{2} F_{2}^{\prime}}{\partial z \partial y}=\frac{\partial^{2} F_{4}^{\prime}}{\partial y \partial x} \\
& \frac{\partial^{2} F_{3}^{\prime}}{\partial t^{\prime} \partial z}=-\frac{\partial^{2} F_{1}^{\prime}}{\partial z \partial y}=-\frac{\partial^{2} F_{2}}{\partial z^{2}}=\frac{\partial^{2} F_{4}^{\prime}}{\partial z \partial x} .
\end{aligned}
$$




$$
\begin{aligned}
& \frac{\partial^{2} F_{4}^{\prime}}{\partial t^{\prime 2}}=\frac{\partial^{2} F_{1}^{\prime}}{\partial t^{\prime} \partial z}=-\frac{\partial^{2} F_{2}^{\prime}}{\partial t^{\prime} \partial y}=-\frac{\partial^{2} F_{3}^{\prime}}{\partial t^{\prime} \partial x} \\
& \frac{\partial^{2} F_{4}^{\prime}}{\partial t^{\prime} \partial x}=\frac{\partial^{2} F_{1}^{\prime}}{\partial x \partial z}=-\frac{\partial^{2} F_{2}}{\partial x \partial y}=-\frac{\partial^{2} F_{3}^{\prime}}{\partial x^{2}} \\
& \frac{\partial^{2} F_{4}^{\prime}}{\partial y \partial t^{\prime}}=\frac{\partial^{2} F_{1}^{\prime}}{\partial y \partial z}=-\frac{\partial^{2} F_{2}}{\partial y^{2}}=-\frac{\partial^{2} F_{3}^{\prime}}{\partial y \partial x} \\
& \frac{\partial^{2} F_{4}^{\prime}}{\partial t^{\prime} \partial z}=\frac{\partial^{2} F_{1}^{\prime}}{\partial z^{2}}=-\frac{\partial^{2} F_{2}^{\prime}}{\partial z \partial y}=-\frac{\partial^{2} F_{3}^{\prime}}{\partial z \partial x} .
\end{aligned}
$$

Note: The functions considered in this paper are of class $C^{2}$. In order to obtain the equations for the electric and gravitational potential in (1) and (2) we will use the sets (29) - (32).

\section{Coupling Equations.}

Analyzing the set of equations (30) and (31) on their first line of equalities, it is possible to obtain connections between gravitational, electric and magnetic potentials. This is only possible because the set of equations (29) and (32) can be used as coupling equations:

In order to discriminate the notation used here, will shall regard $F_{2}$ for $\phi$ and $F_{3}$ for $\varphi$, and that these functions as well as their densities, are respectively gravitational and electrical functions depending on $t^{\prime}, x, y$ and $z$. Thus we have the following equations:

(i) Analysing the set of equations (30):

$$
\begin{gathered}
\frac{\partial^{2} \phi}{\partial t^{\prime 2}}-4 \pi G \rho=-\frac{\partial F_{1}^{\prime}}{\partial t^{\prime} \partial x} \\
\frac{\partial \phi}{\partial x^{2}}=\frac{\partial F_{1}^{\prime}}{\partial t^{\prime} \partial x} \\
-\frac{\partial^{2} \varphi}{\partial t^{\prime} \partial z}=\frac{\partial^{2} F_{4}^{\prime}}{\partial t^{\prime} \partial y} \\
\frac{\partial \varphi}{\partial y \partial x}=-\frac{\partial^{2} F_{4}^{\prime}}{\partial t^{\prime} \partial y}
\end{gathered}
$$

Adding up the sides of the equations above, we get:

$$
\frac{\partial^{2} \phi}{\partial t^{\prime 2}}-4 \pi G \rho+\frac{\partial^{2} \phi}{\partial x^{2}}-\frac{\partial^{2} \varphi}{\partial t^{\prime} \partial z}+\frac{\partial^{2} \varphi}{\partial x \partial y}=0
$$


Making simple changes in the equations we obtain:

$$
\frac{\partial}{\partial t^{\prime}}\left(\frac{\partial \phi}{\partial t^{\prime}}-\frac{\partial \varphi}{\partial z}\right)=\frac{\partial}{\partial x}\left(-\frac{\partial \phi}{\partial x}-\frac{\partial \varphi}{\partial y}\right)+4 \pi G \rho .
$$

or,

$$
\frac{\partial^{2} \phi}{\partial t^{\prime}}-\frac{\partial}{\partial z} \frac{\partial \varphi}{\partial t^{\prime}}=\frac{\partial}{\partial x}\left(-\frac{\partial \phi}{\partial x}-\frac{\partial \varphi}{\partial y}\right)+4 \pi G \rho
$$

but,

$$
\frac{\partial \varphi}{\partial t^{\prime}}=c^{2}(\nabla \cdot \mathbf{A})
$$

where $\mathbf{A}=\left(A_{x}, A_{y}, A_{z}\right)$ is magnetic potential vector. Substituting this last result into equation (35), we have:

$$
\frac{\partial^{2} \phi}{\partial t^{\prime}}+c^{2} \frac{\partial}{\partial z}(\nabla \cdot A)=\frac{\partial}{\partial x}\left(-\frac{\partial \phi}{\partial x}-\frac{\partial \varphi}{\partial y}\right)+4 \pi G \rho
$$

(ii) Now using the set of equations (31), it follows that:

$$
\begin{gathered}
-\frac{1}{c^{2}} \frac{\partial^{2} \varphi}{\partial t^{\prime 2}}(t, x, y, z)+4 \pi \rho_{e}\left(t^{\prime}, x, y, z\right)=-\frac{\partial^{2} F_{1}^{\prime}}{\partial t^{\prime} \partial y} \\
\frac{\partial^{2} \varphi}{\partial y^{2}}=\frac{\partial^{2} F_{1}^{\prime}}{\partial t^{\prime} \partial y} \\
-\frac{\partial^{2} \phi}{\partial t^{\prime} \partial z}=\frac{\partial^{2} F_{4}^{\prime}}{\partial t^{\prime} \partial x} \\
\frac{\partial^{2} \phi}{\partial x \partial t}=-\frac{\partial^{2} F_{4}^{\prime}}{\partial t^{\prime} \partial x}
\end{gathered}
$$

Adding sides of the equations above, we obtain:

$$
-\frac{1}{c^{2}} \frac{\partial^{2} \varphi}{\partial t^{\prime}}+4 \pi \rho_{e}+\frac{\partial^{2} \varphi}{\partial y^{2}}-\frac{\partial^{2} \phi}{\partial t^{\prime} \partial z}+\frac{\partial^{2} \phi}{\partial y \partial x}=0,
$$

or,

$$
\frac{\partial}{\partial t^{\prime}}\left(-\frac{1}{c^{2}} \frac{\partial \varphi}{\partial t^{\prime}}-\frac{\partial \phi}{\partial z}\right)=\frac{\partial}{\partial y}\left(-\frac{\partial \varphi}{\partial y}-\frac{\partial \phi}{\partial x}\right)-4 \pi \rho_{e}
$$

Using again the fact that:

$$
\frac{\partial \varphi}{\partial t^{\prime}}=-c^{2}(\nabla \cdot \mathbf{A})
$$

and substituting in equation (38) generates the following equation:

$$
\frac{\partial}{\partial t^{\prime}}\left(\nabla \cdot \mathbf{A}-\frac{\partial \phi}{\partial z}\right)=\frac{\partial}{\partial y}\left(-\frac{\partial \varphi}{\partial y}-\frac{\partial \phi}{\partial x}\right)-4 \pi \rho_{e}
$$




\section{Concluding Remarks.}

This study enabled us to determine some connections among electric, gravitational and magnetic potential fields. Then, priority was given to temporal equations, considering that the coupled evolution of these fields is of fundamental importance for Physics and also for our further research.

The main results obtained throughout this work can be briefly as:

(i) The magnetic vectorial potential influences the gravitational density;

(ii) The magnetic, gravitational and electrical potential can be obtained through a single mathematical equation.

The coupling exposed here shows a connection that exists between potentials as shown in (36) and (39).

\section{Acknowledgments}

The authors have been partialy supported by FAPEMA, a Scientific Agency of the Maranho State, through research grants.

\section{References}

[1] J.M. Machado, M.F. Borges, New ramarks on the differenciability of hypercomplex functions, International Journal of Pure and Applied Mathematics, 8, No. 1 (2002), 85-101.

[2] J.A.P.F. Marão, M.F. Borges, Geometrical hypercomplex coupling between electric and gravitational fields, International Journal of Pure and Applied Mathematics (2013), To Appear.

[3] J.A.P.F. Marão, M.F. Borges, A note on the hypercomplex RiemannCauchy like relations for quaternions and Laplace equations, International Journal of Pure and Applied Mathematics (2013), To Appear.

[4] Kunihiko Kodaira, Complex Analysis, Cambridge Studies in Advanced Mathematics, Cambridge University Press Cambridge (2007), 406pp. 